\title{
Breast Metastase From Anorectal Melanoma
}

\author{
Rhizlane Belbaraka ${ }^{\mathrm{a}, \mathrm{d}}$, Amal Asabbane ${ }^{\mathrm{b}}$, Hafid Hachic, Brahim Khalil Elgueddari ${ }^{\mathrm{b}}$, \\ Hassan Errihani ${ }^{a}$
}

\begin{abstract}
Breast metastases from extramammary carcinomas are very rare. Primary anorectal melanoma is a rare and aggressive disease with poor prognosis. Despite aggressive surgery, the 5-year survival is less than $10 \%$. Primary anorectal melanoma metastasis to the breast is exceptional. Only two cases were reported in literature. We present a case of breast metastase from primary anorectal melanoma successfully managed with surgery and chemotherapy with longterm free survival. We also briefly reviewed the appropriate literature, emphasizing the diagnostic and therapeutic approaches.
\end{abstract}

Keywords: Breast metastase; Anorectal melanoma

\section{Introduction}

Primary anorectal melanoma is a rare and aggressive disease accounting for approximately $1-3 \%$ of all colorectal and anal cancers [1]. It's an uncommon site of primary melanoma with almost equal male to female ratio and with an average age of presentation between the fifth and the sixth decades of life [1]. Anorectal melanoma occur poor prognosis with high metastatic potential. Breast metastasis from anorectal melanoma is exceptional, only two cases were described in the literature $[2,3]$. We report the third case of metastatic breast from primary anorectal melanoma.

Manuscript accepted for publication June 15, 2010

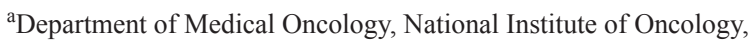
Rabat, Morocco

${ }^{\mathrm{b}}$ Department of Radiothrapy, National Institute of Oncology, Rabat, Morocco

${ }^{\mathrm{c}}$ Department of Surgery, National Institute of Oncology, Rabat,

Morocco

${ }^{\mathrm{d}}$ Corresponding author: Department of Medical Oncology, National Institute of Oncology, Rabat, Morocco. Email: r_belbaraka@yahoo.fr
}

doi:10.4021/jmc2010.06.104e

\section{Case Report}

A 59-year-old woman was admitted to our hospital in March 2007 with a 6-month history of rectal bleeding, tenesmus and weight loss. Physical examination revealed an ulcerative mass about $4 \mathrm{~cm}$ above the anal sphincter, without evidence of invasion in the sphincter. Proctosigmoidoscopy, with multiple biopsies, confirmed digital findings and concluded on anorectal melanoma. Pre-operative CT scan of the chest and abdomen showed no distant metastasis. Serum levels of tumor markers including carcinoembryonic antigen (ACE) and Ca 19-9 were within normal ranges. A typical abdominoperineal resection (APR) was performed. The pathologic stage was III A according to the AJCC TNM classification. No adjuvant treatment was performed. Eighteen months later, the patient presented with 1-month history of a rapidly growing mass in the upper quadrant of the right breast. Physical examination confirmed a 3-cm diameter, firm and not fixed lump. There was no evidence of axillary lymphadenopathy. Mammograms showed a well-defined mass lesion, not obviously malignant (Fig. 1). Core biopsies showed poorly differentiated carcinoma.

Immunohistological control proved to be negative for

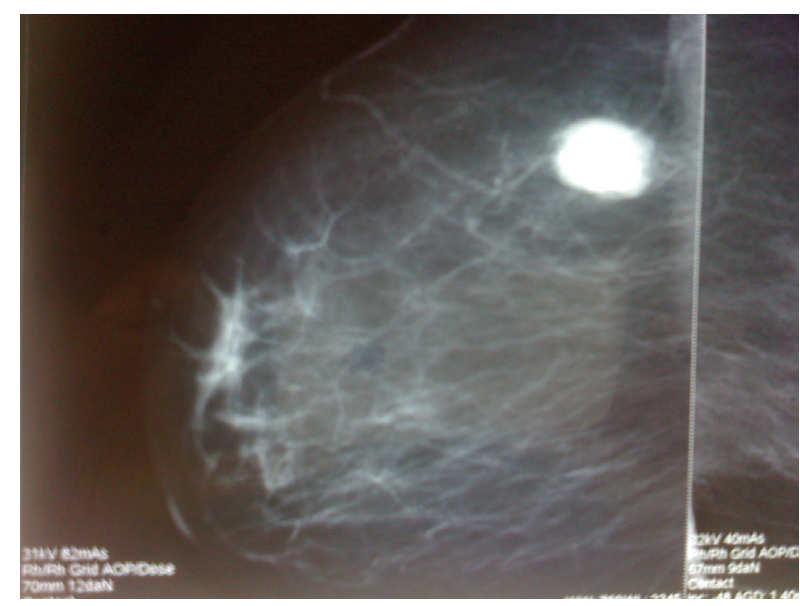

Figure 1. Lateral view mammography showing breast metastasis from anorectal melanoma. 
estrogen receptor, progesterone receptor and HER-2, and revealed positive HMB-45 and EMA. CT scan of the chest, abdomen and pelvis showed no metastatic disease. The patient underwent mastectomy without axillary lymp-node dissection. After surgery, she received Six cycles of dacarbazine chemotherapy regimens. One year after the mastectomy, she is well with no evidence of recurrence.

\section{Discussion}

Anorectal malignant melanoma is an aggressive malignant tumor. Most patients with such melanomas complain for bleeding, pain, or an anal mass.

Metastases occur via lymphatic and hematogenous routes and it has been reported that $38 \%$ of patients have already metastatic disease at the time of diagnosis [4].

Lymphatic spread to mesenteric nodes is more common than to inguinal nodes while lungs, liver and bones are the most frequent sites of distant metastases. Metastases in the breast is very unusual and very rare localization of metastasis in anorectal melanoma. There are only two cases reported in the literature.

Breast metastases from extramammary primary tumors are uncommon, accounting for approximately $1.3-6.6 \%$ of all malignant mammary tumors [5]. Apart from hematopoietic neoplasms, cutaneous malignant melanoma is one of the most common neoplasms with which secondary involvement of the mammary parenchyma is known [6].

Clinical and radiological features mean it is often quite difficult to differentiate between a primary and a metastatic mammary neoplasm. Mammography shows an irregular mass with no calcifications. Correct management of metastatic disease to the breast can prevent unnecessary mutilation.

This rare incidence of metastases to the breast from tumors without any anatomical connection to the breast raises questions about the possible underlying mechanisms for such events.

It seems that breast metastases from malignant melanoma probably occur more frequently in younger than in older women [7]. The predilection for metastatic spread to the breast in premenopausal women may be due to hormonal influences in the translocation of melanoma cells to the breast parenchyma. However, our patient, and also the two cases which were be reported on 1999, concern a post menopausal women $[2,3]$.

The finding of anorectal melanoma that has metastasized to the breast requires complete evaluation for diffuse metastatic disease, because in most cases it is associated with wide dissemination, in particular to the brain, lung, and liver.

It is essential to recognize that malignant tumors in the breast might be metastatic melanomas, so as to avoid un- necessary surgery. It is widely accepted that wide excision is sufficient to control all metastatic tumors of the breast.

It is very important for both surgeon and pathologist to consider the diagnosis of metastatic malignant melanoma. The treatment of metastatic malignant melanoma in the breast is excision of the lesion with clear margins, followed by systemic therapy for the primary disease, obviously after comprehensive evaluation of the staging.

Despite sporadic promising reports, regardless of surgical approach, metastatic anorectal melanoma remains a highly lethal malignancy with five-year survival less than $20 \%$ [8].

To our knowledge, only two cases of anorectal melanomas which have metastasized to the breast were reported in the literature. Our case is unusual not only in that the primary site is the anorectum, but also it was very succefully managed with one year free survival.

\section{Competing Interest}

The authors declare no competing of interest.

\section{References}

1. Klas JV, Rothenberger DA, Wong WD, Madoff RD. Malignant tumors of the anal canal: the spectrum of disease, treatment, and outcomes. Cancer 1999;85(8):16861693.

2. Lee JF, Leung KL, Leow CK, Lau WY. An unusual case of breast metastasis from an anorectal melanoma. Eur J Surg Oncol 1999;25(4):441-442.

3. Ozguroglu M, Ozaras R, Tahan V, Demirkesen C, Demir G, Dogusoy G, Buyukunal E, et al. Anorectal melanoma metastatic to the breast. J Clin Gastroenterol 1999;29(2):197-199.

4. Podnos YD, Tsai NC, Smith D, Ellenhorn JD. Factors affecting survival in patients with anal melanoma. Am Surg 2006;72(10):917-920.

5. Bassi F, Gatti G, Mauri E, Ballardini B, De Pas T, Luini A. Breast metastases from cutaneous malignant melanoma. Breast 2004;13(6):533-535.

6. Vergier B, Trojani M, de Mascarel I, Coindre JM, Le Treut A. Metastases to the breast: differential diagnosis from primary breast carcinoma. J Surg Oncol 1991;48(2):112-116.

7. Arora R, Robinson WA. Breast metastases from malignant melanoma. J Surg Oncol 1992;50(1):27-29.

8. Belli F, Gallino GF, Lo Vullo S, Mariani L, Poiasina E, Leo E. Melanoma of the anorectal region: the experience of the National Cancer Institute of Milano. Eur J Surg Oncol 2009;35(7):757-762. 